\title{
Patient Body Mass Index (BMI) Knowledge in a Rural Primary Care Population
}

\author{
Treah Haggerty, MD, MS, Jun Xiang, MS, MA, and Dana King, MD, MS
}

Introduction: Body mass index (BMI) is a traditional method of measuring obesity and an accepted quality measure in many health systems. However, little is known about how patients' understanding of BMI has progressed/changed in the last several years. The primary purpose of this study was to determine the change in patients' knowledge of BMI and weight-related risk factors. A secondary outcome of this study was to determine the incidence of physician discussion about BMI with the patient.

Methods: We administered an anonymous survey to primary care patients in 33 rural family medicine clinics in 2013 and 2017. The survey included 8 questions regarding BMI knowledge and 5 demographic questions.

Results: A total of 573 patients completed the survey. There were 345 participants from 2013 and 238 from 2017. The sample included more women than men. There was no significant difference in age or proportion of comorbidities across the 2 survey years. Compared with year 2013, more survey participants from year $2017 \mathrm{knew}$ that BMI stood for "body mass index" $(71.1 \%$ vs $61.2 \%, P=.02)$ and understood what medical concerns were related to BMI $(65.5 \%$ vs $50.7 \%, P=.0005)$. The percentages of participants who reported that their doctor ever discussed BMI with them went up from $20.1 \%$ to 28.7\% $(P=.02)$.

Conclusion: In this cross-sectional cohort study, it was found that patients' knowledge about BMI and weight-related risk factors has increased modestly. However, despite the increase in patients' knowledge and discussions about BMI with their primary care physician, there was not a decrease in BMI. Future studies are needed to provide more detailed information about the discussion between patients and providers in relation to the patient's personal weight status. (J Am Board Fam Med 2019;32: 413-417.)

Keywords: Body Composition, Body Mass Index, Cohort Studies, Cross-Sectional Studies, Family Physicians, Health Literacy, Obesity, Primary Health Care, Risk Factors, Rural Health, Surveys and Questionnaires

Body mass index (BMI) is a traditional method of measure of obesity and an accepted quality measure in many health systems. A BMI level in the obesity range is associated with multiple risk factors, including type 2 diabetes, hypertension, and cardio-

This article was externally peer reviewed.

Submitted 24 July 2018; revised 6 January 2019; accepted 9 January 2019.

From West Virginia University School of Medicine, Department of Family Medicine, Morgantown (TH, JX, DK).

Funding: Research reported in this publication was supported by the National Institute of General Medical Sciences of the National Institutes of Health under award number 2U54GM104942-02. The content is solely the responsibility of the authors and does not necessarily represent the official views of the National Institutes of Health.

Conflict of interest: none declared.

Corresponding author: Treah Haggerty, MD, MS, 6040 University Town Center Drive, Morgantown, WV 26501 (E-mail: haggertyt@wvumedicine.org). vascular disease. ${ }^{1}$ Treatment of the obesity-related risk factors increases health care costs and use of resources secondary to the associated morbidity and mortality. ${ }^{2}$ Patient involvement in discussions about weight and weight loss are key to success in obesity management. ${ }^{3}$ However, little is known about how patients' understanding of BMI has progressed/changed in the last several years.

Previous studies have found that primary care patients do not report having discussions with their physicians about weight management. ${ }^{4,5}$ In addition, it has been noted that physicians do not identify patients' weight statuses as overweight or obese. This lack of cross-communication in relation to weight in the clinical office visit has been found to be important to patient activation for weight management improvement. Discussion of 
BMI with a physician or other health care provider is associated with more motivation and greater weight loss. 5

Between 2013 and 2017 there has been an increase in the measurement of BMI at the practicebased level. The National Committee for Quality Assurance uses the Health care Effectiveness Data and Information Set to evaluate performance improvement tools. Through the Health care Effectiveness Data and Information Set, it was found that measurements of BMI ranged from $41.5 \%$ to $89.6 \%$ depending on the insurance plan in 2013 to $67.1 \%$ to $95 \%$ based on insurance plans in $2017 .{ }^{6} \mathrm{It}$ is believed that providers will identify patients at risk of obesity to provide intervention with improving identification of a patient's BMI. The West Virginia Practice-Based Research Network (WVP$\mathrm{BRN}$ ) has also participated in multiple studies related to addressing obesity in the clinical setting through the use of technology and improved patient and provider interactions.

Five years ago, the WVPBRN embarked on a statewide cross-sectional study to determine the status of BMI knowledge and discussions with providers among patients in primary care practices. ${ }^{4}$ Now, 5 years later, the PBRN assessment has been repeated to determine whether knowledge and use of BMI in discussions with primary care providers has become more common and evaluate whether BMI levels changed. We hypothesize that there will be an increase in patient-level BMI knowledge in this 5 -year time frame secondary to an increase in practice improvement activities and quality measurement. The primary purpose of this study was to determine the change in patients' knowledge of $\mathrm{BMI}$ and weight-related risk factors. Secondary outcomes measured included patient knowledge about their own BMI and whether they discussed weight management with their physician.

\section{Methods}

Survey participants were recruited from rural primary care practices in the WVPBRN. The participants represent a sample of convenience at participating PBRN practices and were surveyed in the waiting room while waiting to be seen in June 2013 or June 2017 at participating practices. The surveys were assisted by medical students doing a summer externship in rural family medicine. Sixteen primary care practices in 2013 and 17 in 2017 were contacted by West Virginia University Department of Family Medicine and gave permission to be included in the study. Patients recruited from the participating practices represented a broad geographic representation from the rural West Virginia population. An informal consent was obtained from each survey patient. The study protocol was reviewed and approved by the West Virginia University Health Sciences Institutional Review Board as an exempt study.

Patients were approached in the waiting rooms of the participating practices and invited to take the brief survey if they met the inclusion criteria. The inclusion criteria included being over the age of 18 and proficiency in reading English. Patients with cognitive impairment, had significant visual impairment, or did not report their heights and weights were excluded from the study. There was no penalty for declining to complete the survey. The patients who chose to take the survey were asked to complete the survey and dropped it into a sealed container with a mail slot. All surveys were returned to the Department of Family Medicine and accessed only by the researchers. The surveys were identified by site but not by patient.

\section{Survey}

There are 13 questions in the survey, including 8 questions regarding BMI knowledge and 5 demographic questions. Questions in the survey were reviewed and refined by content experts. The BMI knowledge questions contain both open-end questions, such as "what does BMI stand for" and "A BMI of 25 or greater means...," and "yes or no" questions, such as "Have you heard BMI before?" and "Do you know what your BMI is?" The demographic questions collected information regarding the patient's age, sex, height, weight, and comorbidities, such as hypertension, high cholesterol, obesity, diabetes, and sleep apnea.

\section{Statistical Analysis}

Data from 2013 and 2017 were entered separately and combined later by the same researcher. The 5 open-end questions regarding BMI knowledge were judged "correct" or "incorrect," with 1 point assigned to the correct answer and 0 point assigned to the incorrect answer. A patient would be assigned a total score of 0 to 5 points regarding the numbers of correct answers to the 5 questions. Patient BMI was calculated based on self-reported 
Table 1. Demographic Characteristics of Body Mass Index Survey Patients

\begin{tabular}{|c|c|c|c|}
\hline Characteristic & 2013 Survey $(\mathrm{n}=345)$ & 2017 Survey $(\mathrm{n}=228)$ & $P$ Value* \\
\hline Age (mean \pm standard deviation, years) & $49.6 \pm 16.7$ & $49.0 \pm 18.0$ & 0.79 \\
\hline $\operatorname{Sex}(\%)$ & & & 0.003 \\
\hline Male & 43.0 & 30.7 & \\
\hline Female & 57.0 & 69.3 & \\
\hline $\begin{array}{l}\text { Calculated BMI based on self-reported height } \\
\text { and weight (mean } \pm \text { standard deviation) }\end{array}$ & $29.0 \pm 7.1$ & $30.4 \pm 7.3$ & 0.04 \\
\hline $\begin{array}{l}\text { Weight status based on BMI calculated from } \\
\text { self-reported height and weight (\%) }\end{array}$ & & & 0.09 \\
\hline Under weight $(<18.5)$ & 2.3 & 0 & \\
\hline Normal weight (18.5 to 24.9 ) & 27.0 & 24.0 & \\
\hline Over weight (25 to 29.9 ) & 31.3 & 31.7 & \\
\hline Obese $(\geq 30)$ & 39.4 & 44.3 & \\
\hline \multicolumn{4}{|l|}{$\begin{array}{l}\text { Presence of self-reported comorbid conditions } \\
\quad(\%)\end{array}$} \\
\hline Hypertension & 41.4 & 43.0 & 0.70 \\
\hline Hyperlipidemia & 34.2 & 30.3 & 0.32 \\
\hline Diabetes mellitus & 14.5 & 19.3 & 0.13 \\
\hline Sleep apnea & 9.9 & 12.7 & 0.29 \\
\hline Obesity & 25.8 & 28.5 & 0.48 \\
\hline
\end{tabular}

BMI, body mass index.

*Comparisons of demographic characteristics between the patients in 2013 and the patients in 2017 using Mann-Whitney $U$ test for continuous variables (age and calculated BMI) or $\chi^{2}$ test for categorical variables (sex, BMI group, and presence of comorbid conditions).

heights and weights and categorized into 4 weight groups: underweight (BMI, <18.5), normal weight (18.5 to 24.9 ), overweight (25 to 29.9 ), and obese $(\geq 30)$.

Descriptive statistics were performed for participant demographic information, prevalence of selfreported comorbidities, categorical BMI status, and BMI knowledge. $\chi^{2}$ analysis was used to evaluate the change between the 2 survey years in patients' comorbidities and BMI knowledge based on individual questions. The nonparametric Mann-Whitney $U$ test was used to compare the difference between the 2 survey years in age, calculated BMI, and the total score of BMI knowledge. A $P$ value of less than 0.05 was considered statistically significant for all tests. SAS (version 9.4, 2013, SAS Institute, Inc., Cary, NC) was used for the analysis of this study.

\section{Setting}

Practices were located in the WVPBRN. The WVPBRN is comprised of 94 clinical practice sites located in West Virginia. Each practice site is an outpatient primary care setting.

\section{Results}

A total of 573 patients who completed the BMI survey were included in this study. There were 345 participants from 2013 and 238 from 2017. The average BMI increased from $29.0 \mathrm{~kg} / \mathrm{m}^{2}$ to 30.4 $\mathrm{kg} / \mathrm{m}^{2}$ from 2013 to $2017(P=.04)$. This increase in BMI was consistent with the increase in BMI in West Virginia's population. ${ }^{7}$ There were consistently more female than male participants in both survey years (Table 1). There were no significant differences in the age or the proportion of comorbidities between the 2 survey years.

Table 2 presents the comparison in BMI knowledge between year 2013 and 2017. Compared with year 2013, more survey participants from year 2017 knew that BMI stood for "body mass index" (71.1\% vs $61.2 \%, P=.02)$ and understood what medical concerns were related to BMI $(65.5 \%$ vs $50.7 \%$, $P=.0005)$. Small proportions of participants could correctly define BMI by categories and knew their personal BMI in both cohorts. From year 2013 to 2017, the percentages of participants who had heard of BMI before the survey and reported that their doctor ever discussed BMI with them went up from $63.3 \%$ to $71.4 \%(P=.04)$ and $20.1 \%$ to 
Table 2. Body Mass Index Knowledge of Survey Patients*

\begin{tabular}{lccc}
\hline BMI Knowledge & 2013 Survey & 2017 Survey & $P$ Value \\
\hline What does BMI stand for? & 61.2 & 71.1 & 0.02 \\
BMI is related to what medical concern? & 50.7 & 65.5 & 0.0005 \\
A BMI of 25 or greater means. . & 18.3 & 23.7 & 0.12 \\
A BMI of 30 or greater means. . & 19.2 & 21.1 & 0.57 \\
A BMI of 40 or greater means. . & 14.5 & 20.6 & 0.06 \\
Do you know what your BMI is? & 19.4 & 18.2 & 0.73 \\
Have you heard of BMI before this survey? & 63.3 & 71.4 & 0.04 \\
Has your doctor ever discussed BMI with you & 20.1 & 28.7 & 0.02 \\
\hline
\end{tabular}

BMI, body mass index.

*Data are percentage choosing the correct answer or answering "yes" to the question.

${ }^{\dagger} \chi^{2}$ comparison of responses of BMI knowledge questions between patients from 2013 and patients from 2017.

28.7\% $(P=.02)$, respectively. The total score of BMI knowledge in 2017 was higher than in 2013 (Mann-Whitney $U$ test, $\mathrm{Z}=2.6884, P=.007$ ).

\section{Discussion}

In this cross-sectional cohort study, it was found that patients' knowledge about BMI and weightrelated risk factors has increased modestly. The possible causes are not fully known, although the participants recorded an increase in discussions about BMI with their primary care physician at their clinic visits. Some possible reasons for this increase in BMI knowledge include an increase in BMI measurement as a quality measure across different insurance plans and primary care medical home certification requirements for quality improvement. ${ }^{6}$ Previous literature suggests that physician discussion of healthy lifestyle increases the likelihood that a patient will make healthy lifestyle changes. ${ }^{5}$ However, despite the increase in patients' knowledge and discussions about BMI with their primary care physician, there was not a decrease in BMI in the 2017 study population.

The current findings suggest that further study is needed to produce positive change in the treatment of patients with obesity and produce real change in BMI. Interventions that solely focus on improving weight management knowledge, weight management discussions in the clinic setting, or knowledge of risks associated with obesity may not be as effective as we hoped, that is, participants did not know their personal BMI in either year of the study. It would seem that participants' knowledge of obesity is increasing, but this may not lead to a subsequent change in their own weight status.
Self-reporting weight status and weight management discussions may be a limitation for this study. Previously, it has been found that patients generally underreport their weight status on self-report surveys. ${ }^{8}$ In addition, this study did not take into consideration state and local public health campaigns to increase patient knowledge about obesity. It is not known what has caused the improvement in patient knowledge of obesity factors and BMI. Lastly, another limitation is that the 2013 and 2017 population were different and it is unknown if another factor about the populations could contribute to the study findings.

There are many factors that are correlated with patient behavioral change. Some factors that are related to behavior change include health status, cognitive impairment, age, and education level. Previous studies have shown that improving patient knowledge can be an important step in creating behavioral change. ${ }^{9}$ However, patient knowledge is not the only factor needed to create behavioral change. Patient knowledge needs to be combined with self-efficacy make a behavioral change. ${ }^{10,11}$ This mix of knowledge and self-efficacy can be combined through the use of techniques that actively engage the patient in the discussion of behavioral change, such as shared decision making and motivational interviewing. ${ }^{11,12}$

Improvements in knowledge about BMI and weight-related risk factors could be important, although did not seem to translate into a decrease in BMI. Patients knowing their weight status and the associated risk is an important step for patient activation $^{13}$ in healthy lifestyle factors, but there may be other important steps as well. ${ }^{8}$ Future studies 
may provide more detailed information about the discussion between patients and providers in relation to the patient's personal weight status.

To see this article online, please go to: http://jabfm.org/content/ 32/3/413.full.

\section{References}

1. Must A, Spadano J, Coakley EH, Field AE, Colditz G, Dietz WH. The disease burden associated with overweight and obesity. JAMA1999;282:1523-9.

2. Kim DD, Basu A. Estimating the med care costs of obesity in the United States: systematic review, meta-analysis, and empirical analysis. Value Heal 2016;19:602-13.

3. Laidlaw A, McHale C, Locke H, Cecil J. Talk weight: an observational study of communication about patient weight in primary care consultations. Prim Health Care Res Dev 2015;16:309-15.

4. Post RE, Mendiratta M, Haggerty T, et al. Patient understanding of body mass index (BMI) in primary care practices: a two-state practice-based research (PBR) collaboration. J Am Board Fam Med 2015;28: 475-80.

5. Rose SA, Poynter PS, Anderson JW, Noar SM, Conigliaro J. Physician weight loss advice and patient weight loss behavior change: a literature review and meta-analysis of survey data. Int J Obes 2013; 37:118-28.

6. NCQA. Adult BMI assessment (ABA). Available from: https://www.ncqa.org/hedis/measures/adultbmi-assessment/. Published 2018. Accessed November 27, 2018.
7. Segal LM, Rayburn J, Beck SE. West Virginia State Obesity Data, Rates and Trends - The State of Obesity. Available from: https://stateofobesity.org/ states/wv. Accessed December 4, 2018.

8. Mogre V, Nsoh JA, Wanaba P, Apala P. Demographic factors, weight management behaviours, receipt of healthcare professional's counselling and having knowledge in basic anthropometric measurements associated with underassessment of weight status in overweight and obese type 2 diabetes patients. Obes Res Clin Pract 2016;10:381-9.

9. Ghisi GL de M, Abdallah F, Grace SL, Thomas S, Oh P. A systematic review of patient education in cardiac patients: do they increase knowledge and promote health behavior change? Patient Educ Couns 2014;95:160-74.

10. Äikäs AH, Pronk NP, Hirvensalo MH, Absetz P. Does Implementation follow design? a case study of a workplace health promotion program using the 4-S program design and the PIPE impact metric evaluation models. J Occup Environ Med 2017; 59:752-60.

11. Joseph-Williams N, Elwyn G, Edwards A. Knowledge is not power for patients: A systematic review and thematic synthesis of patient-reported barriers and facilitators to shared decision making. Patient Educ Couns 2014;94:291-309.

12. Elwyn G, Dehlendorf C, Epstein RM, Marrin K, White J, Frosch DL. Shared decision making and motivational interviewing: achieving patient-centered care across the spectrum of health care problems. Ann Fam Med 2014;12:270-5.

13. Truesdale KP, Stevens J. Do the obese know they are obese? N C Med J 2008;69:188-94. 